\title{
Ubiquitin-Conjugating Enzyme 9 Phosphorylation as a Novel Mechanism for Potentiation of the Inflammatory Response
}

\author{
Maria Lauda Tomasi, Komal Ramani, and Minjung Ryoo
}

From the Division of Gastroenterology, Department of Medicine, Cedars-Sinai Medical Center, Los Angeles, California

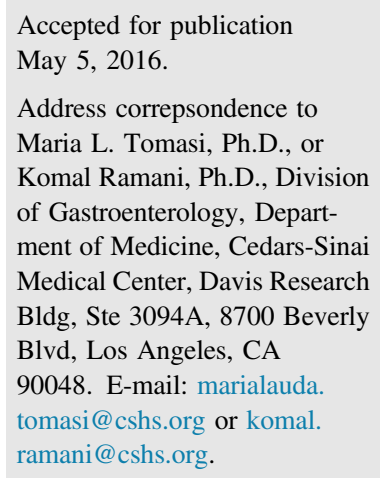

\begin{abstract}
Lipopolysaccharide (LPS), a bacterial endotoxin, induces inflammation in macrophages via activation of $\mathrm{NF}-\kappa \mathrm{B}$ signaling. Sumoylation is a post-translational modification mediated by the small ubiquitin-like modifier, SUMO. Ubiquitin-conjugating enzyme 9 (UBC9) is the only known SUMO conjugating enzyme. LPS treatment lowers SUM0-1 and UBC9 mRNA levels in primary astrocytes. UBC9 can degrade NF- $\kappa B$ inhibitor $\alpha(\mathrm{Ikb} \alpha)$ via a SUM02/3-ubiquitin pathway. However, UBC9 may also promote Ikb $\alpha$ stability by SUM0-1 conjugation that further regulates NF- $\kappa B$ signaling. The role of UBC9 in liver inflammation is unknown. We reported that CDK1-mediated phosphorylation of UBC9 enhanced its stability. Herein, we describe an anti-inflammatory role of UBC9 that is lost when it is phosphorylated during inflammation. LPS exposure caused induction in UBC9 phosphorylation and CDK1 activation specifically in Kupffer cells in vivo and in RAW264.7 macrophages in vitro. Silencing or overexpression experiments in vitro and in vivo showed that UBC9 was required to blunt the proinflammatory response elicited by LPS. LPS stimulation raised the binding of phospho-UBC9 but not the unphosphorylated counterpart, to Ikb $\alpha$ in RAW264.7 macrophages. Hence, phospho-UBC9 may promote NF- $\kappa B$ signaling by regulating Ikb $\alpha$ and this may be a novel mechanism that deregulates liver inflammatory signaling. (Am J Pathol 2016, 186: 2326-2336; http://dx.doi.org/10.1016/j.ajpath.2016.05.007)
\end{abstract}

The gut-derived endotoxin lipopolysaccharide (LPS) that is the component of the Gram-negative bacterium's outer membrane plays a crucial role in alcoholic liver injury. LPS levels are high in the blood of alcoholics and in ethanol-fed rodents. ${ }^{1}$ Kupffer cells become activated in response to elevated levels of LPS during ethanol feeding ${ }^{2}$ and produce immunoregulatory cytokines, such as tumor necrosis factor $\alpha(\mathrm{TNF}-\alpha), \mathrm{IL}-1 \beta, \mathrm{IL}-6$, arachidonic acid, as well as reactive oxygen species. ${ }^{3}$ The NF- $\kappa \mathrm{B}$ proteins play a major role in LPS-mediated inflammatory response by activating the expression of the above cytokine genes. ${ }^{4}$ The activity of $N F-\kappa B$ is regulated by its interaction with the $N F-\kappa B$ inhibitor $\alpha$ protein $(\operatorname{Ikb} \alpha)$ that is known to prevent nuclear translocation of $\mathrm{NF}-\kappa \mathrm{B}$, retaining it in the cytoplasm. ${ }^{4}$

Activation of Kupffer cells appears to modulate liver injury ${ }^{5}$ and is also known to trigger the induction of inflammatory cytokines in hepatocytes. ${ }^{5}$ Hepatocytes respond to LPS-mediated stimulation by inducing the levels of inducible nitric oxide synthase (iNOS). ${ }^{6}$ Kupffer and hepatocyte cell lines such as RAW264.7 and AML12, respectively, show similar profiles of stimulation by LPS as observed in primary cells from mouse liver ${ }^{3,5}$ and are therefore appropriate tools to study mechanisms of LPSmediated cytotoxicity.

Sumoylation is a protein post-translational modification mediated by the ubiquitin-like protein, SUMO. This process controls diverse cellular functions, such as cell cycle, apoptosis, signal transduction pathways, ${ }^{7}$ and production of reactive oxygen species, during an inflammatory response. ${ }^{8}$ Sumoylation itself is induced by oxidative stress. ${ }^{8}$ Vertebrate systems express four distinct SUMO genes (SUMOI to $S U M O 4$ ) encoding small proteins approximately $12 \mathrm{kDa}$ in size that are moderately related to ubiquitin. ${ }^{8}$ SUMO modification of target proteins occurs via a multiple step process similar to ubiquitination but involving

Supported by NIH grant 5 K01 AA022372-04 (M.L.T.).

Disclosures: None declared. 
SUMO-specific E1 (SUMO-activating SAE1/SAE2), E2 [SUMO or ubiquitin-conjugating enzyme 9 (UBC9)], and E3 (SUMO-ligating) enzyme family. ${ }^{7,8}$

UBC9 is the sole SUMO-conjugating enzyme. ${ }^{9}$ Dual roles of UBC9 are known in the regulation of inflammatory signals via modulation of Ikb $\alpha$. UBC9-mediated heterologous addition of SUMO-2/3 and ubiquitin to Ikb $\alpha$ promote efficient degradation of this protein by the $26 \mathrm{~S}$ proteasome pathway, leading to enhanced NF- $\kappa \mathrm{B}$ activation during a TNF- $\alpha-$ mediated inflammatory response. ${ }^{7}$ In normal cells as well as TNF- $\alpha$-stimulated cells, modified forms of IkB $\alpha$ that have SUMO-1 modification have been described. ${ }^{10}$ This SUMO-1 sumoylation is mediated by UBC 9 and renders $\mathrm{IkB} \alpha$ resistant to degradation by the proteasome pathway and further prevents NF- $\mathrm{B}$-dependent transcription. ${ }^{10}$

Oxidative stress resulting in increased reactive oxygen species production is known to be a stimulus for protein sumoylation. $^{7}$ LPS treatment lowers SUMO and UBC9 mRNA levels in primary astrocytes, ${ }^{11}$ but the mechanisms are unclear. SUMO-1 and UBC9 overexpression decreases NOS2 (iNOS) promoter activity and suppresses proinflammatory response in astrocytes. ${ }^{11}$ Conversely, increased nitric oxide caused global hyposumoylation in mammalian cells $^{12}$; however, the mechanism is unknown. How UBC9 is regulated during LPS signaling in the liver is unknown so far. The goal of this study is to evaluate how LPS toxicity regulates UBC9 and the mechanism of anti-inflammatory action of UBC9 in the liver. We recently published that UBC9 was a substrate for phosphorylation mediated by cyclin-dependent kinase 1 (CDK1) and phospho-UBC9 exhibited enhanced protein stability. ${ }^{13}$ Herein, we describe the role of UBC9 in controlling inflammation. We show that lack of $U B C 9$ potentiates LPS toxicity, whereas forced expression of $U B C 9$ suppresses it. LPS signaling is known to cause early changes in phosphorylation and activation of mitogen-activated protein kinases. ${ }^{14}$ We have identified a novel mechanism by which LPS induces phosphorylation of UBC9 specifically in Kupffer cells and not in hepatocytes. This phosphorylation event allows UBC9 to interact with negative regulators of inflammation (Ikb $\alpha$ ) and may play an important role in reversing UBC9's anti-inflammatory function during LPS toxicity.

\section{Materials and Methods}

\section{Cell Lines}

RAW264.7 mouse macrophage cell line and AML12 mouse hepatocyte cell line were purchased from ATCC (Manassas, VA). C57BL/6 mice were purchased from Jackson Laboratories (Bar Harbor, ME) and housed at the Cedars-Sinai Medical Center vivarium facility. LPS was purchased from Sigma (St. Louis, MO). UBC9 siRNA and negative control siRNAs were purchased from Shanghai Gene Pharma Company (Shanghai, China). UBC9-HA tagged expression vector was from Origene (Rockville, MD). Transfection reagents,
LipofectamineRNAiMAX for siRNA and Lipofectamine 2000 for plasmid transfection, were purchased from Invitrogen (Carlsbad, CA).

\section{LPS or UBC9 siRNA Injections in C57BL/6 Mice and Liver/Cell Isolations}

Three-month-old C57BL/6 mice were injected i.p. for 4 hours with $15 \mathrm{mg} / \mathrm{kg}$ body weight of LPS dissolved in saline. Control mice were injected in the same way with saline alone. Mice were injected i.p. with $100 \mu \mathrm{g}$ of $U B C 9$ or negative control siRNA complexed to $50 \mu \mathrm{g}$ lipid-based in vivo transfection reagent (Altogen Biosystems, Las Vegas, NV). After the above treatment periods, mice were sacrificed and livers were collected and preserved for RNA or protein isolation, as described previously. ${ }^{13}$ The core services provided by the Liver Center at Cedars-Sinai were used for the isolation of hepatocytes and Kupffer cells from LPS-treated or control mice, as previously described. ${ }^{15}$ Simultaneous isolation of mouse hepatocytes and Kupffer cells is described briefly below. Mouse liver was perfused through the superior vena cava with Eagle's minimal essential medium at $5 \mathrm{~mL} /$ minute for 10 minutes, and then with digestion buffer [Dulbecco's modified Eagles' medium (DMEM) containing $0.044 \%$ (w/v) collagenase; Sigma] for 6 to 10 minutes. The liver was agitated in a rotary shaker for 10 to 15 minutes to further digest and dissociate the cells in DMEM containing $10 \mu \mathrm{g} / \mathrm{mL}$ DNase I. The cell suspension was centrifuged at $700 \mathrm{rpm}(50 \times g)$ for 1 minute under $4^{\circ} \mathrm{C}$. The resulting cell pellet was resuspended in $10 \mathrm{~mL}$ of Hanks buffer mixed with $5 \mathrm{~mL}$ Percoll (Sigma P1644), and centrifuged at $150 \times g$ for 5 minutes at $4^{\circ} \mathrm{C}$. The cell pellet was washed two times with DMEM medium and centrifuged at $700 \mathrm{rpm}(50 \times g)$. The supernatant containing nonparenchymal cells was centrifuged at $150 \times g$ for 5 minutes, and the pellet was washed twice with DMEM medium. The final cell suspension was laid on the top of four OptiPrepTM gradients (1.085, $1.058,1.043,1.034)$ and centrifuged in the SW-41Ti rotor at $50,000 \times g$ for 15 minutes at $25^{\circ} \mathrm{C}$. Kupffer cells were isolated from the 1.043/1.058 interface. The viability of both cell types was checked by trypan blue exclusion.

All animal procedures were performed by established protocols approved by the Institutional Animal Care and Use Committee of the Cedars-Sinai Medical Center that has been American Association of Laboratory Animal Care accredited since 1967. Animals were treated humanely and all procedures were in compliance with the institution's guidelines for the use of laboratory animals.

\section{RAW264.7 and AML12 Cell Culture and LPS Treatment}

RAW264.7 cells were grown in DMEM containing 10\% serum and antibiotics at a density of $1 \times 10^{6}$ cells in $10-\mathrm{cm}$ dishes or $0.4 \times 10^{6}$ in 6 -well plates. AML12 cells were grown in DMEM-F12 medium containing a cocktail of insulin, selenium, and transferrin (ThermoScientific, 
Rockford, IL) and $0.05 \mu \mathrm{g} / \mathrm{mL}$ dexamethasone (Sigma). Cells were treated with LPS at a final concentration of 100 $\mathrm{ng} / \mathrm{mL}$ for 4 hours. After the incubation period, RNA or protein was isolated for various assays described below.

\section{Transient Transfection Assays}

RAW264.7 cells $\left(0.4 \times 10^{6}\right.$ cells per well $)$ were reverse transfected 2 nMUBC9 siRNA complexed to RNAiMAX for 12 hours. For apoptosis assays, $20 \mathrm{nmol} / \mathrm{L} U B C 9$ siRNA was transfected into cells for 24 hours. One microgram of UBC9-HA tagged vector or empty vector control was transfected into $0.4 \times 10^{6}$ cells for 24 hours using the Lipofectamine 2000 reagent.

\section{Quantitative RT-PCR}

Total RNA from total liver, Kupffer cells, hepatocytes, or RAW264.7 cells was reverse transcribed using M-MLV RT (Takara Biosciences/Clontech, Mountain View, CA) and was subjected to quantitative RT-PCR using TaqMan probes for mouse UBC9TNF- $\alpha$, IL-10, iNOS, IL-6, and the housekeeping gene, GAPDH (ABI, Foster City, CA). ${ }^{16}$ The thermal profile consisted of initial denaturation at $95^{\circ} \mathrm{C}$ for 3 minutes followed by 45 cycles at $95^{\circ} \mathrm{C}$ for 3 seconds and at $60^{\circ} \mathrm{C}$ for 30 seconds. The cycle threshold ( $\mathrm{Ct}$ value) of the target genes was normalized to that of GAPDH to obtain the $\Delta \mathrm{Ct}$. The $\Delta \mathrm{Ct}$ was used to find the relative expression of target genes according to the formula: Relative expression $=2-\Delta \Delta \mathrm{Ct}$,
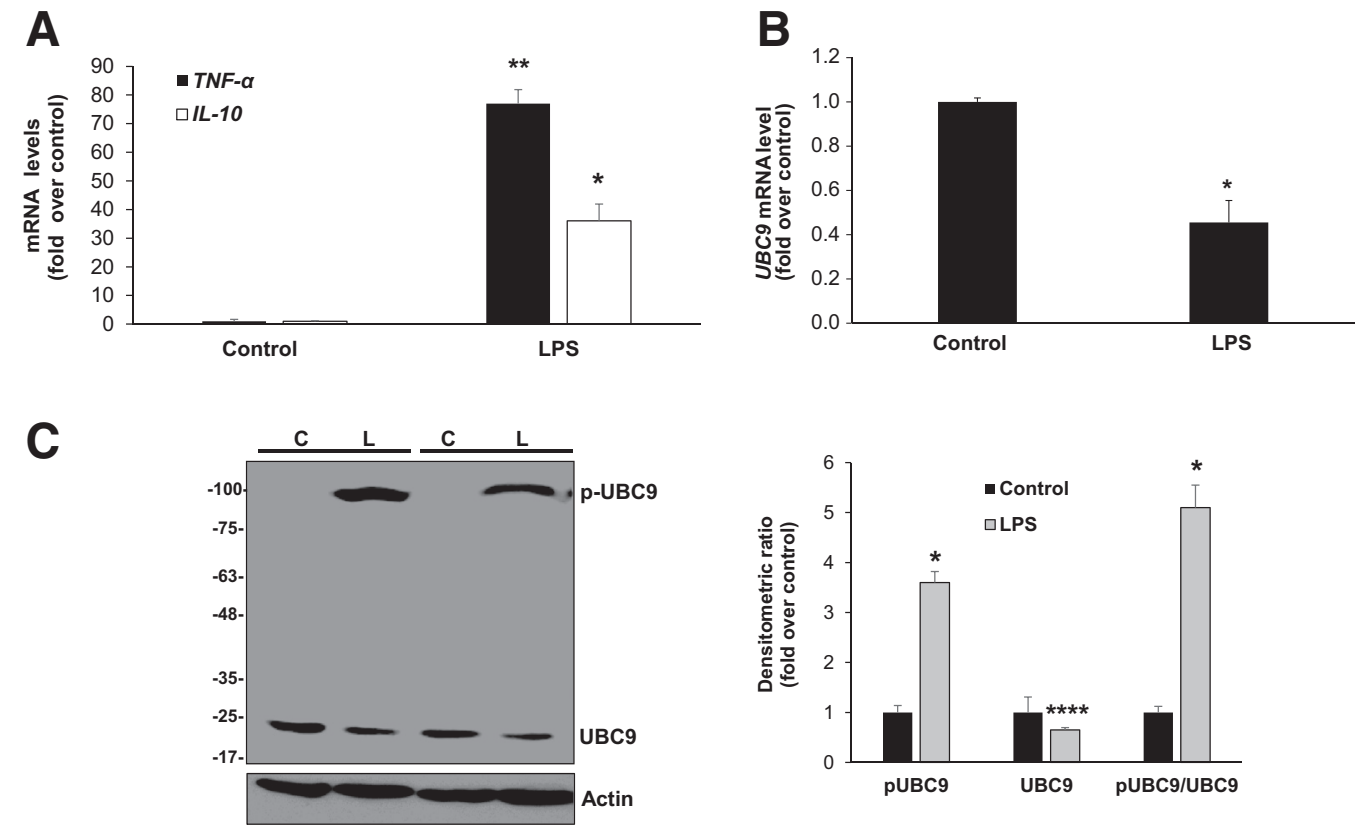

Figure 1 Expression and phosphorylation of ubiquitin-conjugating enzyme 9 (UBC9) in mice livers injected with lipopolysaccharide (LPS). Total RNA or protein was extracted from control or LPS-treated mouse livers, as described in Materials and Methods. A: The mRNA levels of tumor necrosis factor (TNF)- $\alpha$ and IL-10 were estimated by relative quantitative PCR. B: The mRNA level of UBC9 was estimated by relative quantitative PCR. C: Total liver protein was subjected to phosTag SDS-PAGE as described under experimental section. The densitometric ratio of phosphorylated and unphosphorylated bands is represented as fold over control. Data are expressed as means \pm SEM from seven animal groups $(\mathbf{A}-\mathbf{C}) . n=2$ representative groups. ${ }^{*} P<0.05,{ }^{* *} P<0.01$, and ${ }^{* * * * P}<0.001$ versus control. C, control; L, lipopolysaccharide. 
A

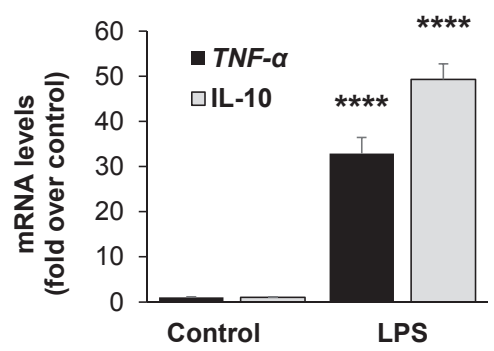

C

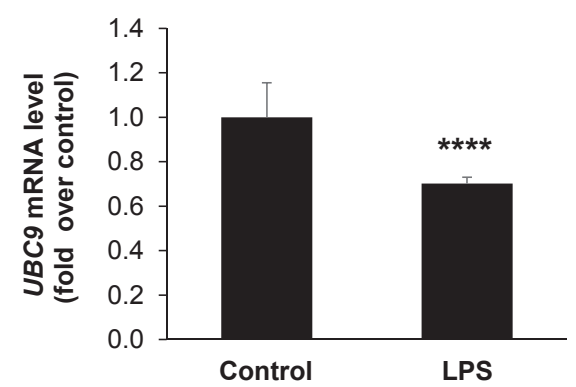

D
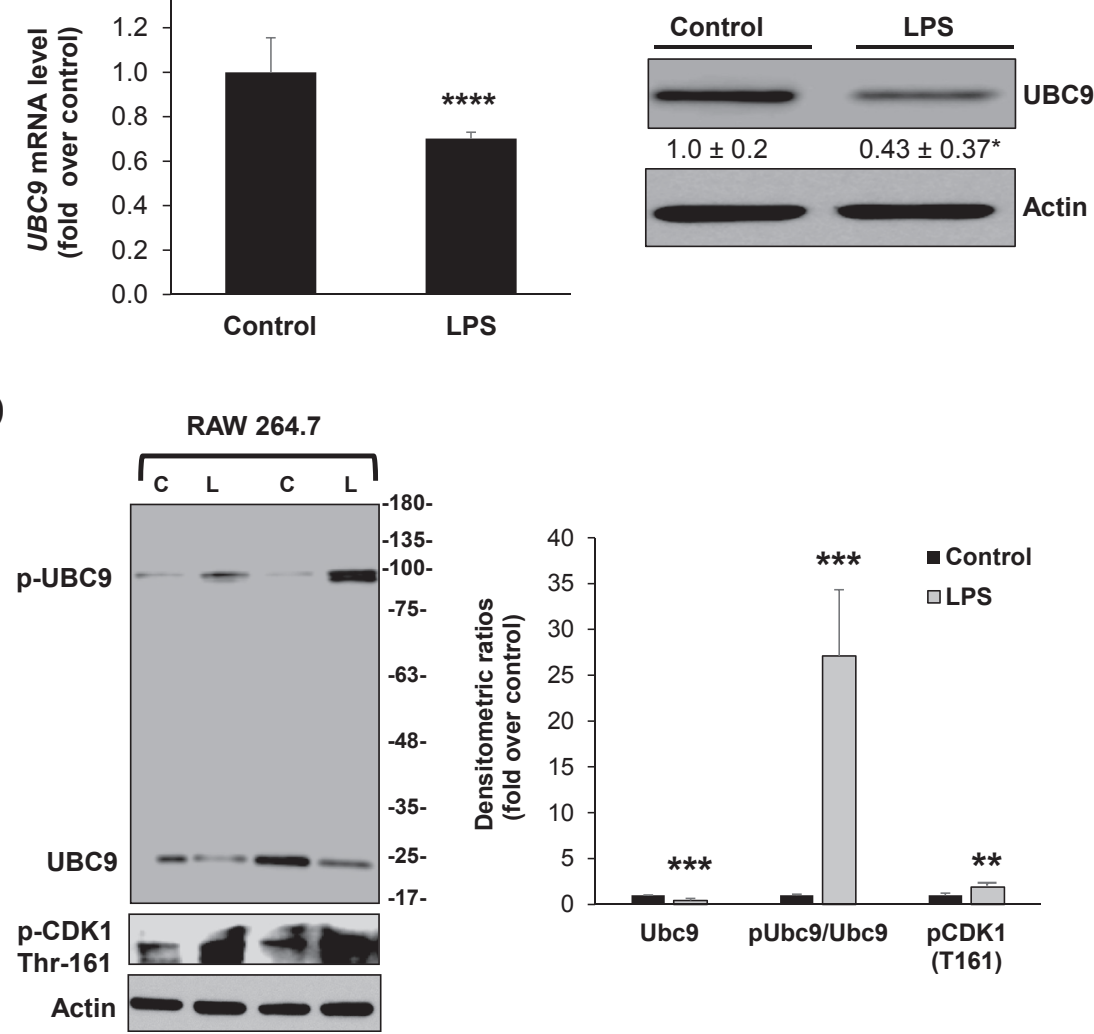

Figure 2 Expression and phosphorylation of ubiquitin-conjugating enzyme 9 (UBC9) in RAW264.7 macrophage cell line treated with lipopolysaccharide (LPS). A: Total mRNA levels of tumor necrosis factor (TNF)- $\alpha$ and IL-10 were compared between control and LPS-treated cells by relative quantitative PCR. B: TNF- $\alpha$ release into the medium of RAW264.7 cells was measured by enzyme-linked immunosorbent assay, as described in Materials and Methods. C: Total mRNA for UBC9 was measured by quantitative PCR and UBC9 protein was checked by Western blotting. D: Control or LPStreated RAW264.7 protein extracts were subjected to phosTag SDS-PAGE, as described in Materials and Methods. The densitometric ratio of phosphorylated and unphosphorylated bands is represented as fold over control. The p-CDK1 Thr-161 was measured by Western blotting using phospho-specific antibodies. Data are expressed as means \pm SEM from six experimental groups (A-D). Two representative experiments are shown. ${ }^{*} P<0.05,{ }^{*} * P<0.01$, $* * * P<0.005$, and $* * * * P<0.001$ versus control. $C$, control; L, lipopolysaccharide.

\section{TNF- $\alpha$ Enzyme-Linked Immunosorbent Assay}

The release of TNF- $\alpha$ in the medium of RAW264.7 cells on LPS stimulation was estimated by the mouse TNF- $\alpha$ ELISA Ready-Set-Goaffymetrix reagent (eBiosciences, San Diego, CA) using immobilized TNF- $\alpha$ antibody in a 96-well format. The concentration of TNF- $\alpha$ was measured from a standard curve of absorbance (A450 nm) of known TNF- $\alpha$ standards versus their concentrations in picograms per $\mathrm{mL}$ $(\mathrm{pg} / \mathrm{mL})$ as per the instructions from the manufacturer.

\section{Phospho-Affinity Purification Chromatography and Coimmunoprecipitation/Immunoblotting Assays}

Total protein from control or LPS-treated RAW264.7 cells was loaded onto a PhosphoCruz phospho-protein affinity purification system column (Santa Cruz Biotechnology, Dallas, TX) and phosphorylated proteins (column-bound) were separated from unphosphorylated proteins (column flow through) according to the manufacturer's instructions. Equal amounts of phosphorylated or unphosphorylated protein fractions were immunoprecipitated with two micrograms of UBC9 antibody, according to previously published protocols, ${ }^{13,17}$ and then immunoblotted with Ikb $\alpha$ antibody.

\section{Apoptosis Measurement by Hoechst Staining}

RAW264.7 cells were grown in 6-well dishes and apoptosis in negative control or $U B C 9$ siRNA-treated cells was measured at different times by incubation of cells with Hoechst 33342 stain (Sigma) according to a previously described protocol. ${ }^{18}$

\section{Statistical Analysis}

Data are represented as means \pm SEM from six experimental groups. Statistical analysis was performed using analysis of variance followed by Student's $t$-test. Significance was defined as $P<0.05$. For animal studies involving 
A

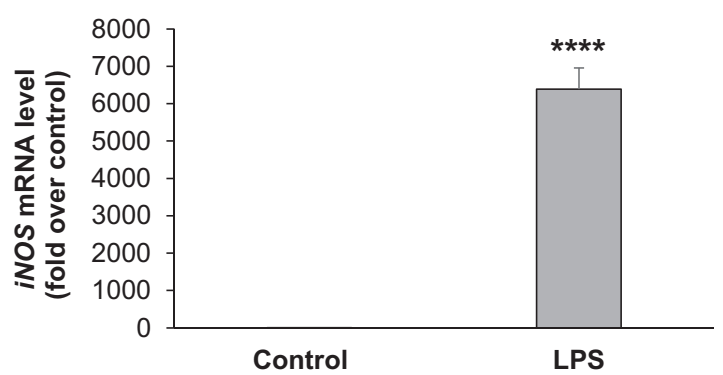

B
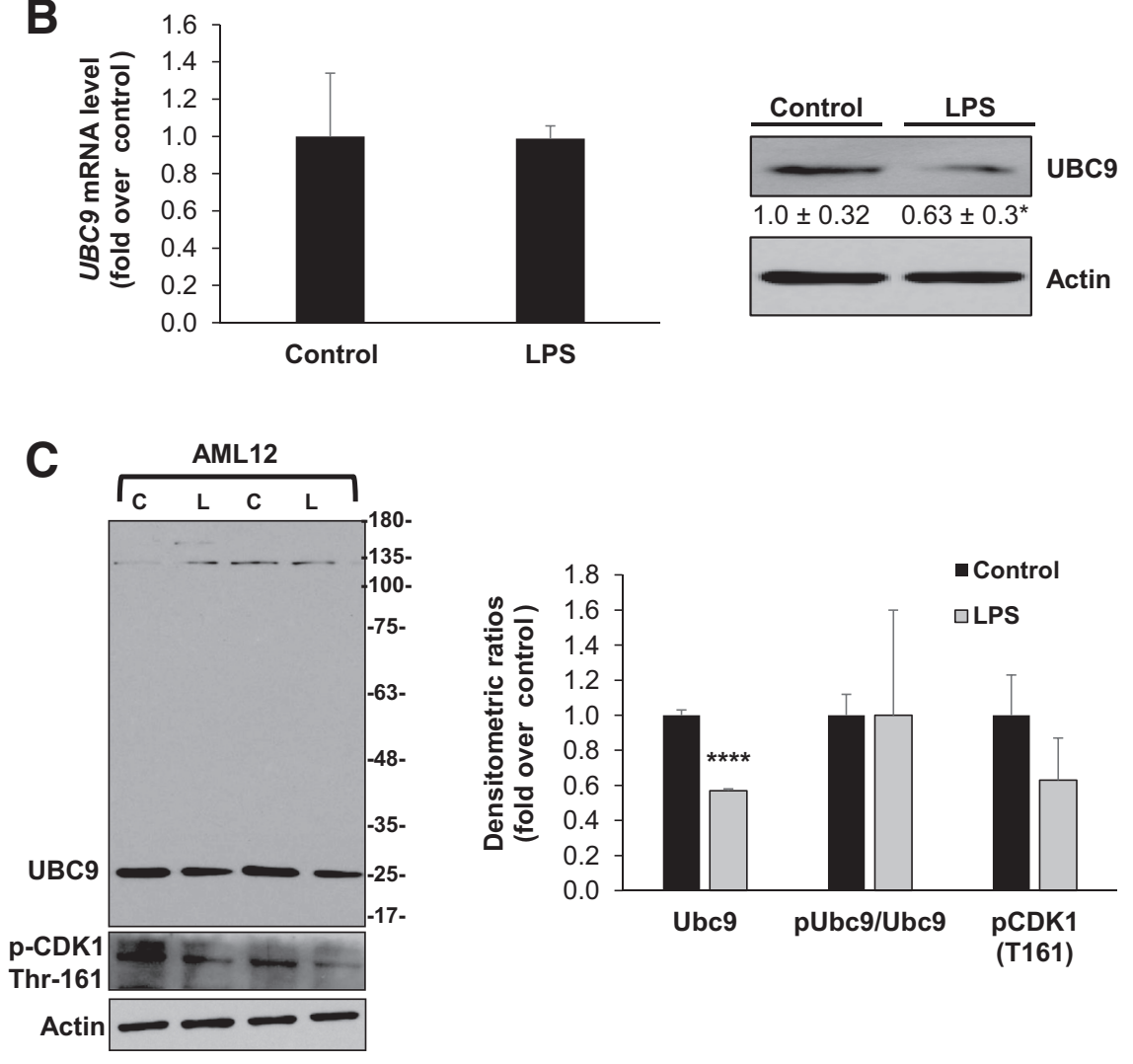

Figure 3 Expression and phosphorylation of ubiquitin-conjugating enzyme 9 (UBC9) in AML12 hepatocyte cell line treated with lipopolysaccharide (LPS). A: The mRNA level of iNOS was compared between control and LPS-treated cells by quantitative PCR. B: UBC9 mRNA was measured by quantitative PCR and UBC9 protein was checked by Western blotting. C: Control or LPS-treated AML12 protein extracts were subjected to phosTag SDS-PAGE, as described in Materials and Methods. The p-CDK1 Thr-161 was measured by Western blotting using phosphospecific antibodies. Data are expressed as means \pm SEM from six experimental groups. Two experiments are shown. ${ }^{*} P<0.05,{ }^{* * *} P<0.01$ versus control. C, control; L, lipopolysaccharide.
LPS injection or UBC9 siRNA injection, number of animals used is based on previously reported experimental designs and the power of hypothesis test to achieve statistical significance. For each in vivo experiment, statistical analysis has been performed using the open access online sample size calculator (Simple Interactive Statistical Analysis, http://www.quantitativeskills.com/sisa/calculations/power.htm; last accessed March 2, 2016) to determine the number of mice to be used to achieve statistical significance using a statistical power of 0.8 and a significance level of 0.05 .

\section{Results}

Expression and Phosphorylation of UBC9 in Livers of Mice Injected with LPS

LPS injection in mice raised the inflammatory markers, TNF- $\alpha$ and IL-10 by 40 and 70 folds, respectively, in the liver compared to control livers (Figure 1A). LPS inhibited the expression of UBC9 mRNA by $60 \%$ compared to control (Figure 1B). LPS stimulation also enhanced the phosphorylation of UBC9 as detected by phosTag-bound phospho-UBC9 compared to a decrease in the lower molecular weight unphosphorylated counterpart (Figure 1C). The phospho/total ratio of UBC9 was fivefold higher in LPS samples compared to controls (Figure 1C).

\section{Expression and Phosphorylation Profile of UBC9 in RAW264.7 Cells}

LPS stimulated the expression of inflammatory cytokine markers, TNF- $\alpha$ and IL-10 mRNA (Figure 2A) and TNF- $\alpha$ release into the medium of RAW264.7 cells (Figure 2B), consistent with previous reports. ${ }^{3}$ The LPS response was associated with a $40 \%$ to $60 \%$ decrease in both the mRNA and protein levels of UBC9 (Figure 2C). LPS treatment 

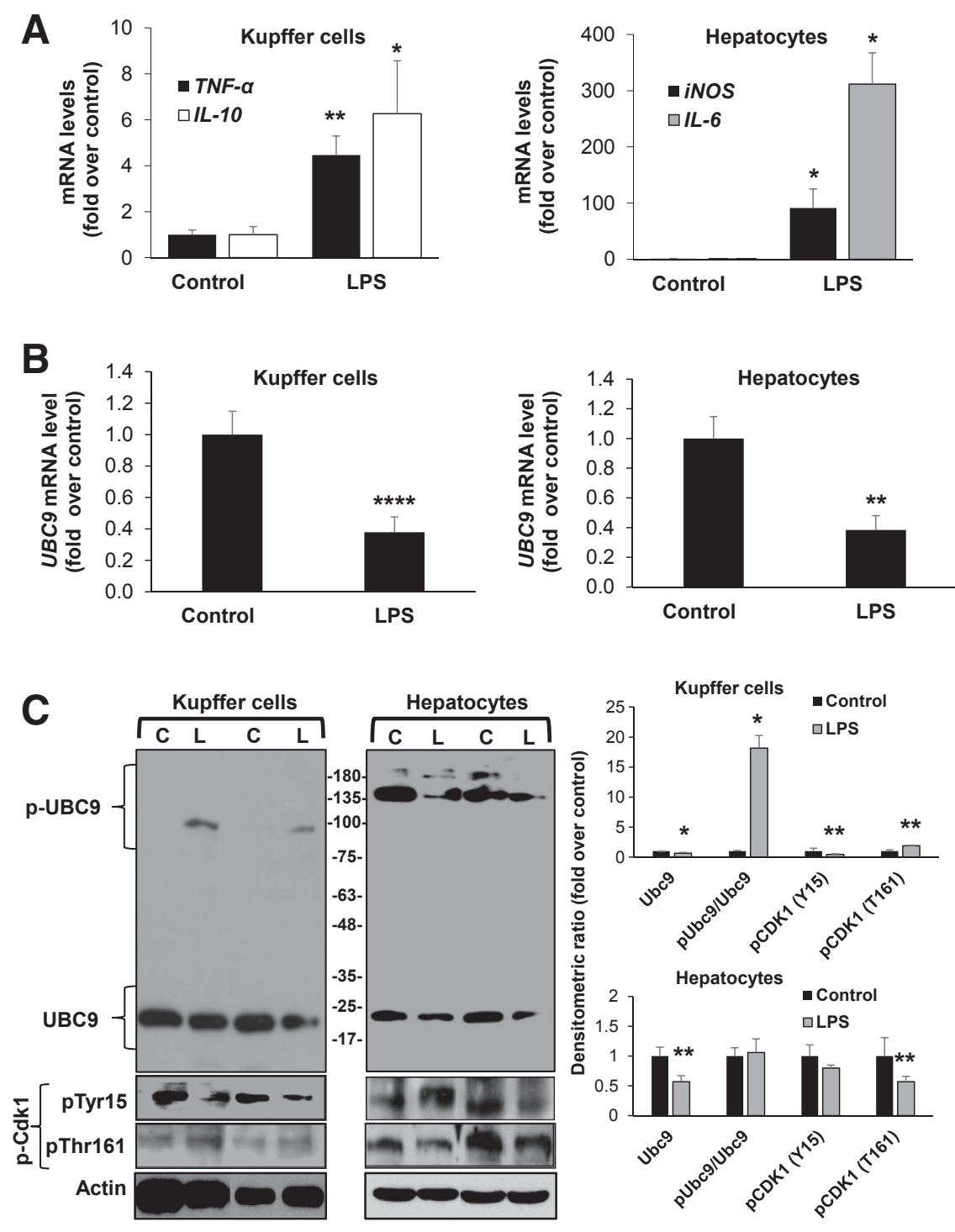

Figure 4 Expression and phosphorylation of ubiquitin-conjugating enzyme 9 (UBC9) in primary Kupffer cells and hepatocytes isolated from lipopolysaccharide (LPS)-treated mouse livers. Kupffer cells and hepatocytes were isolated from control or LPS-treated mouse livers as described in Materials and Methods. A: The mRNA levels of inflammatory markers, tumor necrosis factor (TNF)- $\alpha$ or IL-10 (Kupffer cells) and inducible nitric oxide synthase (iNOS) or IL-6 (hepatocytes) were measured by quantitative PCR. B: Relative UBC9 mRNA levels were estimated by quantitative PCR in Kupffer cells or hepatocytes. C: Total protein from Kupffer cells or hepatocytes was subjected to phosTag Western blotting to estimate UBC9 phosphorylation. pCDK1 Tyr-15 or Thr-161 phosphorylation was performed by Western blotting using phospho-specific antibodies. Two experiments are shown. Data are expressed as means \pm SEM from six cell isolations $(\mathbf{A}-\mathbf{C})$. ${ }^{*} P<0.05,{ }^{* *} P<0.01$, and ${ }^{* * * *} P<0.001$ versus control. C, control; L, lipopolysaccharide. raised the phosphorylation of UBC9 compared to a decrease in the unphosphorylated counterpart (Figure 2D). The phospho/total ratio of UBC9 was 27-fold higher in LPS-treated RAW264.7 cells compared to controls (Figure 2D). Increased UBC9 phosphorylation was associated with a twofold increase in the activity of CDK1 (phosphorylation at Thr-161) in LPS samples compared to control (Figure 2D).

\section{Expression and Phosphorylation Profile of UBC9 in AML12 Cells}

LPS treatment of AML12 cells raised the level of iNOS mRNA (Figure 3A) as reported previously. ${ }^{6}$ AML12 cells exhibited a $40 \%$ decrease in UBC9 protein levels without a corresponding change in UBC9 mRNA (Figure 2B). The phosphorylation of UBC9 protein was not clearly detectable on a phosTag gel. A faint signal of phosphorylated form was detected in AML12 cells but the molecular weight was different from that seen in RAW264.7 cells, indicating that the phosphorylation profile and phospho-sites of UBC9 in AML12 cells may be different from RAW264.7 cells (Figure 2D and Figure 3C). Also, there was significant variability in the amount of this band between different control or LPS-treated preparations (Figure 3C). However, as with total UBC9 (Figure 3B), unphosphorylated UBC9 was also decreased on LPS stimulation (Figure 3C). Consistent with the lack of enhanced phosphorylation, hepatocytes exhibited a decrease in CDK1 activity on LPS stimulation (Figure 3C).

\section{Expression and Phosphorylation of UBC9 in Kupffer Cells and Hepatocytes from LPS-Treated Livers}

As evidenced previously, ${ }^{3,6}$ we confirmed that LPS stimulated the expression of inflammatory cytokine markers, TNF- $\alpha$ and IL-10 mRNA in Kupffer cells (Figure 4A) and iNOS and IL-6 mRNA in hepatocytes from LPS-treated 

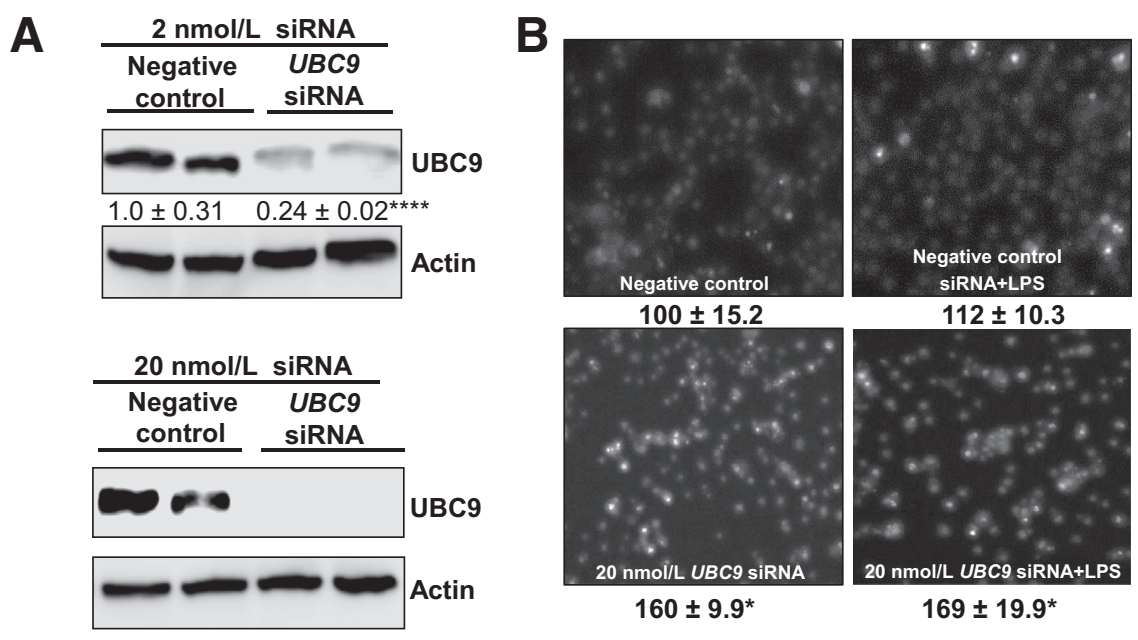

C

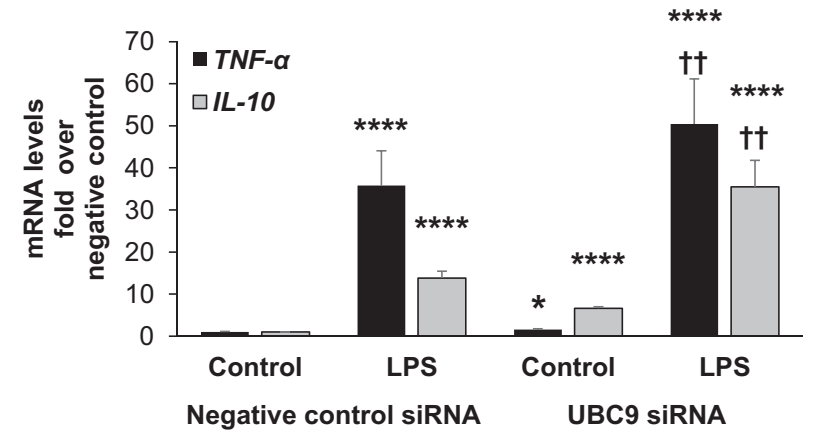

D

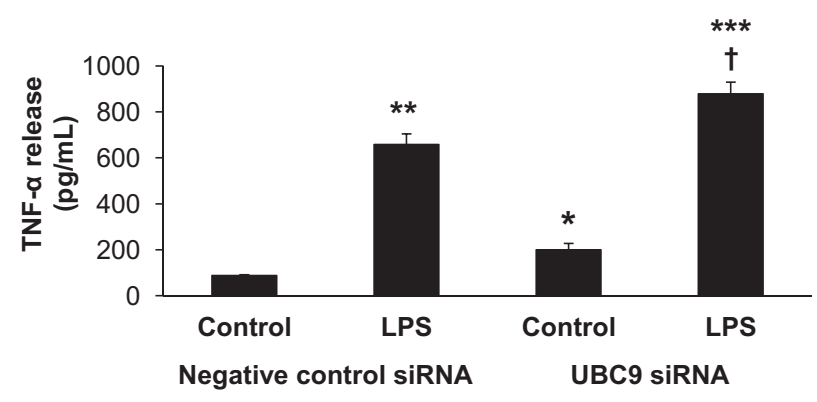

Figure 5 Effect of ubiquitin-conjugating enzyme 9 (UBC9) silencing on the lipopolysaccharide (LPS)-mediated inflammatory response in RAW264.7 cells. A: Knockdown efficiency of UBC9 SiRNA (2 or $20 \mathrm{nmol} / \mathrm{L}$ dose) compared to a negative control siRNA was measured by Western blotting of UBC9 protein. For the $20 \mathrm{nmol} / \mathrm{L}$ dose densitometry was not performed because the protein amount in UBC9 siRNA samples was low and could not be quantitated accurately compared to baseline. B: Cells were treated as in A with 20 nmol/L siRNA for 24 hours and processed for measurement of apoptosis by Hoechst staining, as described in Materials and Methods. C: RAW264.7 cells transfected with a negative control or UBC9 siRNA were treated with or without LPS, as described in Materials and Methods. The expression of tumor necrosis factor (TNF)- $\alpha$ or IL-10 mRNA was measured by quantitative PCR. D: TNF- $\alpha$ release into the medium of RAW264.7 cells was measured by enzyme-linked immunosorbent assay, as described in Materials and Methods. Data are expressed as means \pm SEM from six experiments (A-D). ${ }^{*} P<0.05,{ }^{* *} P<0.01$, $* * * P<0.005$, and $* * * * P<0.001$ versus negative control; ${ }^{\dagger} P<0.05,{ }^{\dagger \dagger} P<0.01$ versus LPS treatment. Original magnification, $\times 40(B)$.

mice compared to controls (Figure 4A). LPS stimulation caused a 60\% inhibition in UBC9 mRNA levels in Kupffer cells (Figure 4B) or hepatocytes (Figure 4B). In Kupffer cells, LPS treatment raised the phosphorylation of UBC9 in a phosTag gel and lowered the unphosphorylated form of UBC9, thereby enhancing the phospho/total ratio by 21-fold compared to controls (Figure 4C). In contrast to Kupffer cells, hepatocytes did not show any change in UBC9 phosphorylation on LPS stimulation (Figure 4C). We have previously demonstrated that recombinant UBC9 is phosphorylated by CDK1. ${ }^{13}$ Herein, we show that LPS stimulation raises the activity of CDK1 in Kupffer cells but not hepatocytes by lowering phosphorylation at the inhibitory phosphorylation site, Tyr-15 of CDK $1^{19}$ (Figure 4C) and concomitantly enhancing the Thr-161 phosphorylation that is known to activate CDK $1^{19}$ (Figure 4C). Hence, during LPS stimulation, enhanced CDK1 activation was associated with increased UBC9 phosphorylation specifically in Kupffer cells.

\section{Effect of UBC9 Modulation on the LPS-Mediated Inflammatory Response in RAW264.7 Cells}

Because LPS affected UBC9 phosphorylation specifically in Kupffer cells, we sought to examine whether modulating UBC9 levels in the RAW264.7 cell line could alter LPSmediated inflammatory signals. Knockdown of $U B C 9$ with $2 \mathrm{nmol} / \mathrm{L}$ siRNA for 12 hours caused a $76 \%$ reduction in UBC9 protein in RAW264.7 cells (Figure 5A) without influencing growth or apoptosis (data not shown). Complete UBC9 silencing with $20 \mathrm{nmol} / \mathrm{L}$ siRNA for 24 hours (Figure 5A) increased apoptosis in normal or LPS-treated RAW264.7 cells by 1.6-fold compared to a negative control siRNA (Figure 5B). For further analysis, the $2 \mathrm{nmol} / \mathrm{L}$ 

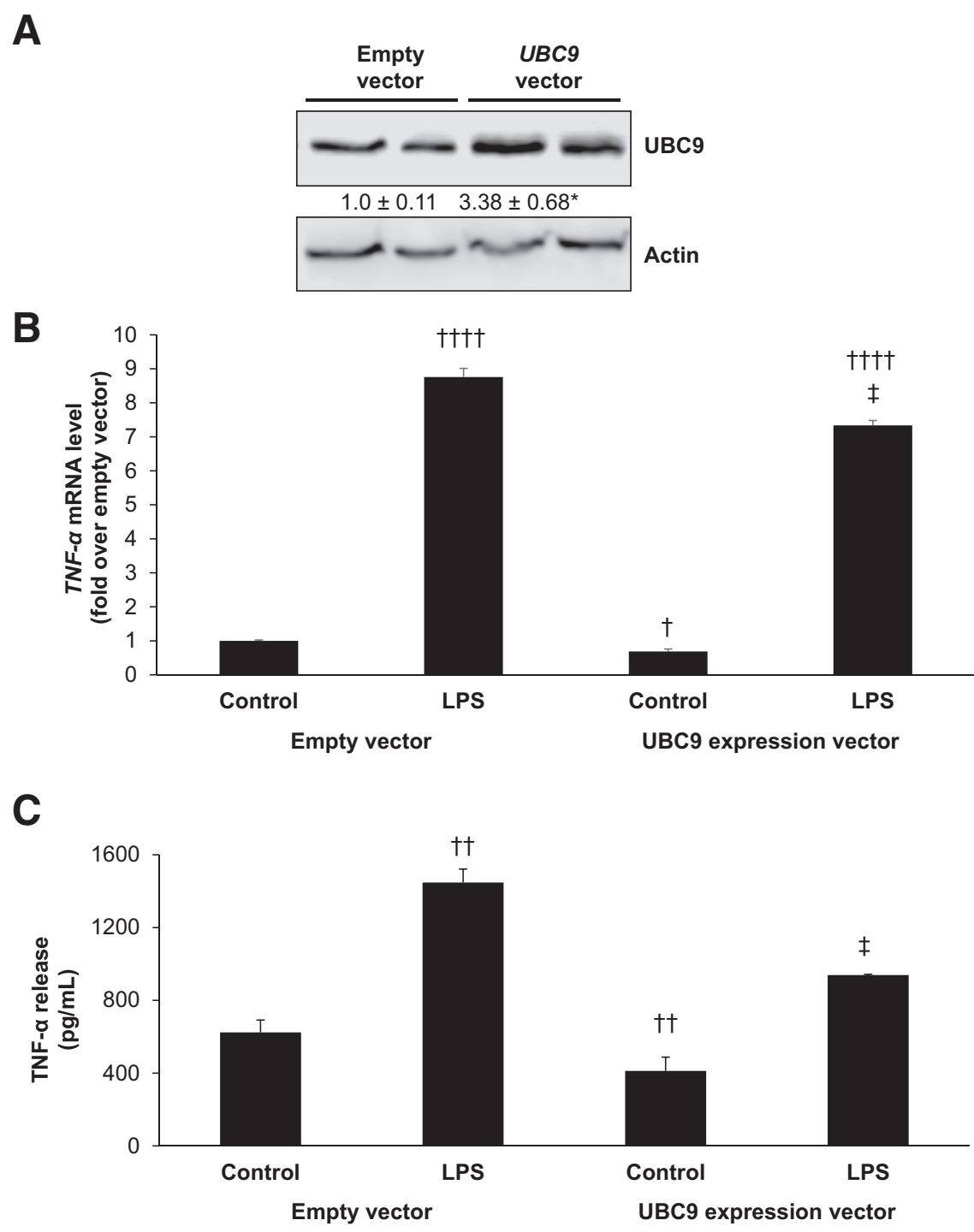

Figure 6 Effect of ubiquitin-conjugating enzyme 9 (UBC9) overexpression on the lipopolysaccharide (LPS)-mediated inflammatory response in RAW264.7 cells. A: Overexpression of UBC9 vector compared to empty vector was determined by Western blotting of UBC9 protein. B: RAW264.7 cells transfected with empty vector or UBC9 vector were treated with or without LPS, as described in Materials and Methods. The expression of tumor necrosis factor (TNF)- $\alpha$ mRNA was measured by quantitative PCR. C: TNF- $\alpha$ release into the medium of RAW264.7 cells was measured by enzyme-linked immunosorbent assay, as described in Materials and Methods. Data are expressed as means \pm SEM from six experiments $(\mathbf{A}-\mathbf{C})$. ${ }^{\star} P<0.05$ versus control; ${ }^{\dagger} P<0.05,{ }^{\dagger \dagger} P<0.01$, and ${ }^{\dagger \dagger \dagger \dagger} P<0.001$ versus empty vector; ${ }^{\ddagger} P<0.05$ versus LPS treatment.
siRNA dose was used. UBC9 knockdown increased the expression of TNF- $\alpha$ (1.5-fold) and IL-10 (6.6-fold) compared to a negative control siRNA (Figure 5C). LPS treatment caused a 30-fold induction in TNF- $\alpha$ and a 14-fold induction of IL-10 compared to control (Figure 5C). UBC9 depletion in RAW264.7 cells further exacerbated the LPS-mediated inflammatory response by causing a 50-fold induction of TNF- $\alpha$ and a 35-fold induction of IL-10 compared to controls (Figure 5C). UBC9 siRNA + LPS combination caused a 2- to 2.5-fold increase in inflammatory response compared to LPS alone (Figure 5C). UBC9 depletion significantly enhanced LPSmediated TNF- $\alpha$ release into the medium of RAW264.7 cells compared to LPS treatment alone (Figure 5D). Forced expression of a $U B C 9$ vector (Figure 6A) into RAW264.7 cells lowered TNF- $\alpha$ mRNA levels by $30 \%$ compared to empty vector controls (Figure 6B). UBC9 vector coincubated with LPS lowered TNF- $\alpha$ mRNA by $25 \%$ (Figure 6B) and TNF- $\alpha$ release by $35 \%$ compared to LPS alone (Figure 6C).
Effect of in Vivo Silencing of UBC9 on Inflammatory Response in the Liver

Direct injection of $U B C 9$ siRNA in mice inhibited $U B C 9$ mRNA levels by $50 \%$ in the liver compared to negative control siRNA-injected liver (Figure 7). Knockdown of UBC9 enhanced TNF- $\alpha$ mRNA levels (1.6-fold) and IL-10 mRNA levels (twofold) compared to a negative control siRNA (Figure 7).

\section{Mechanism of Action of Phospho-UBC9 in LPS Inflammatory Signaling}

It is known that SUMO2/3-ubiquitination and UBC9 are involved in the degradation of $\mathrm{Ikb} \alpha$, a regulator of $\mathrm{NF}-\kappa \mathrm{B}$ that retains it in the cytoplasm, thereby preventing $\mathrm{NF}-\kappa \mathrm{B}$ nuclear translocation and activation of LPS-responsive genes. ${ }^{7}$ Because LPS strongly enhanced UBC9 phosphorylation, we examined whether the phospho-form of UBC9 played a role in promoting the inflammatory signals 


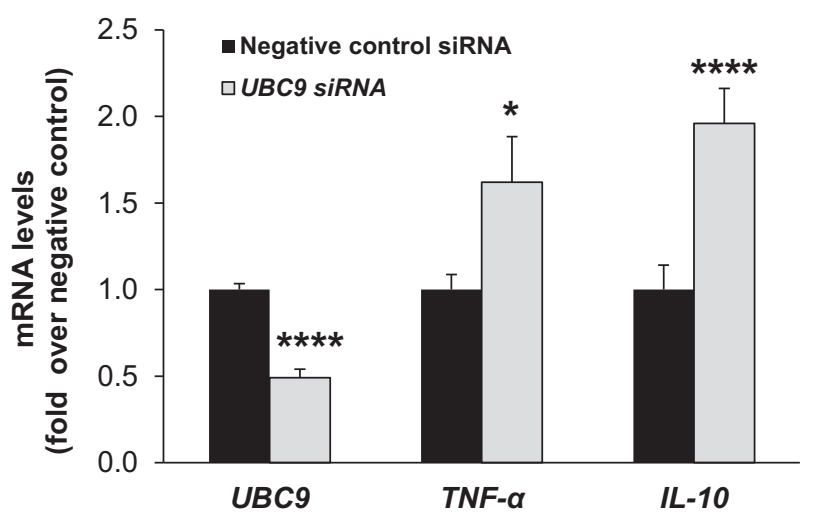

Figure 7 Effect of in vivo silencing of ubiquitin-conjugating enzyme 9 (UBC9) on inflammatory response in the liver. UBC9 siRNA or negative control siRNA was injected into mice, as described in Materials and Methods. Total liver mRNA was subjected to quantitative PCR to measure UBC9, tumor necrosis factor (TNF)- $\alpha$, or IL-10 levels. Data are expressed as means \pm SEM from six animal groups. ${ }^{*} P<0.05,{ }^{*} * * P<0.001$ versus negative control.

mediated by LPS. Our results showed that in normal RAW264.7 cells weak binding of phospho-UBC9 is observed to Ikb $\alpha$. On LPS stimulation, phospho-UBC9 but not unphosphorylated protein, was bound strongly to Ikb $\alpha$ in RAW264.7 cells (sevenfold induction compared to control) (Figure 8A). LPS stimulation reduced both the total levels of UBC9 and Ikb $\alpha$ by $50 \%$ compared to control (Figure 8A). These results suggest that sequestration of Ikb $\alpha$ by phosphorylated UBC9 might be a mechanism by which LPS enhances the inflammatory signal by releasing NF- $\kappa \mathrm{B}$ for induction of TNF- $\alpha$ and IL-10. Not only did LPS lower UBC9, it concomitantly triggered the up-regulation of a phospho-modified UBC9 that had proinflammatory effects on cells. We also confirmed further that any signal that lowered UBC9 in RAW264.7 cells raised the levels of this phosphorylated form. Our results showed that reduction in UBC9 protein levels by siRNA significantly raised the phospho-modified UBC9 protein as detected on a phostag gel (Figure 8B). The findings and proposed scheme of events are summarized in Figure 8C.

\section{Discussion}

The bacterial endotoxin LPS mediates severe inflammatory responses in the liver. Kupffer cells respond to LPS stimulation by exhibiting NF- $\mathrm{B}$-dependent expression of proinflammatory cytokines, such as TNF- $\alpha$ and IL-10. ${ }^{3}$ It is also well established that regulators of NF- $\kappa \mathrm{B}$, the Ikb $\alpha$ proteins are degraded rapidly via the proteasomal pathway during inflammation leading to enhanced shuttling of $\mathrm{NF}-\kappa \mathrm{B}$ to the nucleus favoring cytokine gene expression. ${ }^{4}$ Cells do have potent mechanisms for stabilizing Ikb $\alpha$ such as UBC9-mediated SUMO-1 conjugation of this protein that prevents its degradation. ${ }^{10}$ However, inflammatory signal buildup in cells can overcome this stabilization effect.
Because UBC9 is known to mediate both stabilization (SUMO-1 conjugation) $^{10}$ and degradation of $\mathrm{Ikb} \alpha$ (SUMO2/3-ubiquitin conjugation) $^{7}$ and Kupffer cells are important sites of action of LPS, we examined the molecular mechanism by which UBC9 could modulate inflammatory signals in the liver.

Previously published work has shown that LPS inhibits UBC9 mRNA levels in primary astrocytes. ${ }^{11}$ Our findings in Kupffer cells and hepatocytes that UBC9 mRNA and protein are inhibited during LPS exposure are consistent with previous reports. ${ }^{16}$ Our results also show that UBC9 is an anti-inflammatory molecule because reduction in its amount exacerbates the basal or LPS-stimulated inflammatory signal, whereas overexpression blunts this response both in vitro and in vivo. Complete silencing of UBC9 in RAW264.7 cells led to cell apoptosis, suggesting that UBC9 is required for cell survival. This is consistent with previous literature that demonstrates the importance of $U B C 9$ in the survival of postimplantation mouse embryos ${ }^{20}$ and in regulating apoptosis during stem cell survival and tumorigenesis. ${ }^{21,22}$ We performed controlled silencing of UBC9 (dose of $2 \mathrm{nmol} / \mathrm{L}$ for 12 hours) to prevent apoptosis and to maintain the low UBC9 protein level after knockdown in a range similar to that caused by LPS exposure. For the first time, we have reported in this work that the LPS-mediated down-regulation of UBC9 is associated with a strong induction in phosphorylation of this protein specifically in Kupffer cells but not in hepatocytes. Further support for this result comes from our finding that the activity of CDK1 kinase (pThr-161 phosphorylation) is induced only in Kupffer cells but not in hepatocytes after LPS exposure. We previously reported that CDK1 could phosphorylate recombinant UBC9 and UBC9 phosphorylation enhanced its stability. ${ }^{13}$ Blocking dephosphorylation of UBC9 in cells by phosphatase inhibitors also enhanced the total and phospho-level of UBC9 and stabilized it. ${ }^{13}$ Our results that both the activity of CDK1 and UBC9 phosphorylation are up-regulated in LPS-treated Kupffer cells are in sync with our previously published work. ${ }^{15}$

Because UBC9 normally prevents inflammation and LPS lowered UBC9 along with an induction of phospho-UBC9, we hypothesized that the phospho-UBC9 molecule might be involved in mediating the proinflammatory effects of LPS in Kupffer cells. LPS is known to influence phosphorylation of key players such as mitogen-activated protein kinases during inflammation. ${ }^{14}$ It is also well established that the $\mathrm{Ikb} \alpha$ protein is degraded by UBC9-facilitated SUMO2/ 3 -ubiquitin chain conjugation during inflammation. ${ }^{7}$ Hence, we examined the possibility that phospho-modified UBC9 may interact with Ikb $\alpha$ in Kupffer cells and possibly facilitate its degradation. Our results clearly demonstrate that phospho-UBC9, but not its unphosphorylated counterpart, was able to preferentially bind to Ikb $\alpha$ in LPS-treated RAW264.7 cells. This finding supports our hypothesis that phospho-UBC9 is the major form of UBC9 that interacts with Ikb $\alpha$ in RAW264.7 cells and might play a role in 
A

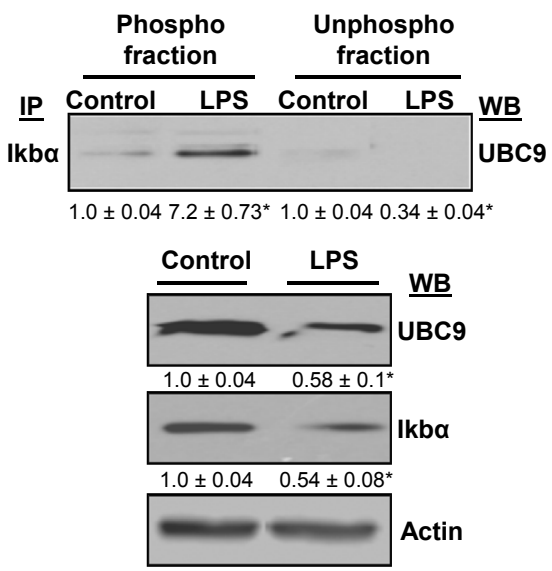

B
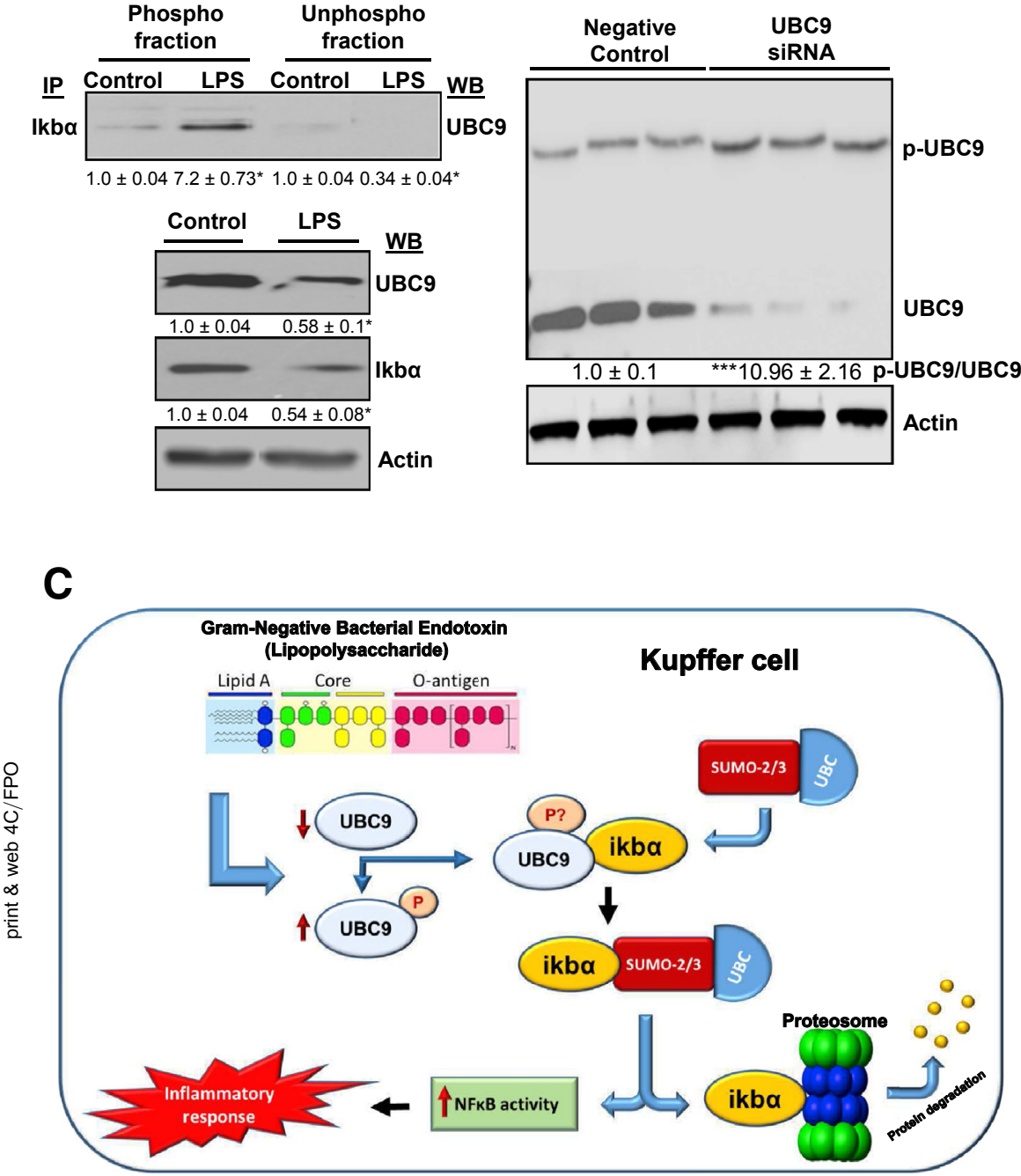

Figure 8 Mechanism of action of phosphoubiquitin-conjugating enzyme 9 (UBC9) in lipopolysaccharide (LPS) inflammatory signaling. A: Total protein from control or LPS-treated RAW264.7 cells separated on phospho-purification column was immunoprecipitated with Ikb $\alpha$ antibody and immunoblotted with UBC9 antibody (left side of panel). Total expression of UBC9, Ikb $\alpha$, and actin (control) was determined by Western blotting (WB; right side of panel). B: Cell extracts from RAW264.7 cells treated with negative control or $2 \mathrm{nmol} / \mathrm{L}$ UBC9 siRNA for 12 hours were subjected to Phostag analysis. Three individual experiments are shown. Densitometric data are represented as a fold of phospho/total ratio of UBC9 siRNA versus control. C: LPS toxicity in Kupffer cells leads to a decrease in UBC9 along with an induction of phospho-UBC9 levels. Phospho-UBC9 exhibits enhanced binding to Ikba. The proposed scheme is that phosphoUBC9 mediates SUM02/3-ubiquitin conjugation and/or lowers SUM0-1 conjugation of Ikb $\alpha$, thereby facilitating Ikb $\alpha$ degradation and enhancing NF- $\mathrm{KB}$ activity enhanced inflammatory reaction. Data are expressed as means \pm SEM from six experiments (A and $\mathbf{B}) .{ }^{*} P<0.05,{ }^{* * *} P<0.005$ versus negative control. IP, immunoprecipitation; $P$, phospho group; UBC, ubiquitin. facilitating Ikb $\alpha$ degradation previously reported by other groups. $^{7}$ Our analysis also shows that both LPS treatment and controlled silencing of UBC9 trigger a compensatory induction of phospho-UBC9. The results reiterate the importance of having a balance of UBC9 levels in the cells. Depletion of UBC9 reserves may drive the cells toward inflammatory signaling because of accumulation of phospho-UBC9 and severe lack of UBC9 is proapoptotic for cells. LPS exerts its inflammatory effect in Kupffer cells by lowering UBC9 levels and concomitantly enhancing the levels of a phospho-modified UBC9 that is more stable ${ }^{13}$ and is active in stimulating the inflammatory signal. We previously showed that CDK1 can phosphorylate UBC9. ${ }^{13}$ On the basis of this result, we hypothesized that LPSmediated UBC9 phosphorylation could be via the activity of CDK1. Indeed in Kupffer cells, LPS treatment enhanced the Thr-161-CDK1 active form, whereas it inhibited the Tyr-15 inactive form. However, in hepatocytes there was decreased activity of CDK1 on LPS exposure and also lower UBC9 levels. Even though the hepatocytes do respond to
LPS stimulation, the mechanism by which UBC9 is phosphorylated in these cells appears to be different from Kupffer cells because this does not seem to be via CDK1 activity. Hence, the phosphorylation events in hepatocytes do not seem to stabilize the protein because a decrease in unphosphorylated protein does not lead to enhanced phospho-forms. The fact that UBC9 phosphorylation and CDK1 activity were specifically induced in Kupffer cells but not in hepatocytes points toward a cell-specific phenomenon that could be targeted to prevent the inflammatory response caused by LPS toxicity.

The next step of this work is to delineate the phosphorylation sites of UBC9 that mediate its binding to Ikb $\alpha$. We believe that this phosphorylation event favors Ikb $\alpha$ degradation as opposed to its stabilization mediated by SUMO-1 conjugation. ${ }^{10}$ To our knowledge, this is the first report of a post-translational modification in UBC9 that favors proinflammatory signaling in the liver. Maintenance of UBC9 level and regulation of its phosphorylation appear to be key mechanisms that define the role of this protein as a guardian 
against inflammation. Further detailed analysis of UBC9 phospho-modification would pave the way for targeting inflammation as a therapeutic strategy for liver disease.

\section{Acknowledgment}

We thank Dr. Shelly Lu (Director of the Liver Research Group at Cedars-Sinai) for providing the necessary infrastructure and support for this work.

\section{References}

1. Fujimoto M, Uemura M, Nakatani $Y$, Tsujita S, Hoppo K, Tamagawa T, Kitano H, Kikukawa M, Ann T, Ishii Y, Kojima H, Sakurai S, Tanaka R, Namisaki T, Noguchi R, Higashino T, Kikuchi E, Nishimura K, Takaya A, Fukui H: Plasma endotoxin and serum cytokine levels in patients with alcoholic hepatitis: relation to severity of liver disturbance. Alcohol Clin Exp Res 2000, 24:48S-54S

2. Cao Q, Mak KM, Lieber CS: Cytochrome P4502E1 primes macrophages to increase TNF-alpha production in response to lipopolysaccharide. Am J Physiol Gastrointest Liver Physiol 2005, 289:G95-G107

3. Kishore R, McMullen MR, Cocuzzi E, Nagy LE: Lipopolysaccharidemediated signal transduction: stabilization of TNF-alpha mRNA contributes to increased lipopolysaccharide-stimulated TNF-alpha production by Kupffer cells after chronic ethanol feeding. Comp Hepatol 2004, 3:S31

4. Tashiro K, Pando MP, Kanegae Y, Wamsley PM, Inoue S, Verma IM: Direct involvement of the ubiquitin-conjugating enzyme Ubc9/Hus5 in the degradation of IkappaBalpha. Proc Natl Acad Sci U S A 1997, 94 : 7862-7867

5. Kanuri G, Bergheim I: In vitro and in vivo models of non-alcoholic fatty liver disease (NAFLD). Int J Mol Sci 2013, 14:11963-11980

6. Vos TA, Van Goor H, Tuyt L, De Jager-Krikken A, Leuvenink R, Kuipers F, Jansen PL: Expression of inducible nitric oxide synthase in endotoxemic rat hepatocytes is dependent on the cellular glutathione status. Hepatology 1999, 29:421-426

7. Aillet F, Lopitz-Otsoa F, Egaña I, Hjerpe R, Fraser P, Hay RT, Rodriguez MS, Lang V: Heterologous SUMO-2/3-ubiquitin chains optimize I $\kappa \mathrm{B} \alpha$ degradation and NF- $\kappa \mathrm{B}$ activity. PLoS One 2012, 7: e51672

8. Pandey D, Chen F, Patel A, Wang CY, Dimitropoulou C, Patel VS, Rudic RD, Stepp DW, Fulton DJ: SUMO1 negatively regulates reactive oxygen species production from NADPH oxidases. Arterioscler Thromb Vasc Biol 2011, 31:1634-1642
9. Gareau JR, Lima CD: The SUMO pathway: emerging mechanisms that shape specificity, conjugation and recognition. Nat Rev Mol Cell Biol 2010, 11:861-871

10. Desterro JM, Rodriguez MS, Hay RT: SUMO-1 modification of IkappaBalpha inhibits NF-kappaB activation. Mol Cell 1998, 2: 233-239

11. Akar CA, Feinstein DL: Modulation of inducible nitric oxide synthase expression by sumoylation. J Neuroinflammation 2009, 26:12

12. Qu J, Liu GH, Wu K, Han P, Wang P, Li J, Zhang X, Chen C: Nitric oxide destabilizes Pias3 and regulates sumoylation. PLoS One 2007, 2 : e1085

13. Tomasi ML, Tomasi I, Ramani K, Pascale RM, Xu J, Giordano P, Mato JM, Lu SC: S-adenosyl methionine regulates ubiquitinconjugating enzyme 9 protein expression and sumoylation in murine liver and human cancers. Hepatology 2012, 56:982-993

14. Guha M, O'Connell MA, Pawlinski R, Hollis A, McGovern P, Yan SF, Stern D, Mackman N: Lipopolysaccharide activation of the MEKERK1/2 pathway in human monocytic cells mediates tissue factor and tumor necrosis factor alpha expression by inducing Elk-1 phosphorylation and Egr-1 expression. Blood 2001, 98:1429-1439

15. Tomasi ML, Ramani K, Lopitz-Otsoa F, Rodríguez MS, Li TW, Ko K, Yang H, Bardag-Gorce F, Iglesias-Ara A, Feo F, Pascale MR, Mato JM, Lu SC: S-adenosylmethionine regulates dual-specificity mitogen-activated protein kinase phosphatase expression in mouse and human hepatocytes. Hepatology 2010, 51:2152-2161

16. Ramani K, Tomasi ML: Transcriptional regulation of methionine adenosyltransferase $2 \mathrm{~A}$ by peroxisome proliferator-activated receptors in rat hepatic stellate cells. Hepatology 2012, 55:1942-1953

17. Ramani K, Donoyan S, Tomasi ML, Park S: Role of methionine adenosyltransferase $\alpha 2$ and $\beta$ phosphorylation and stabilization in human hepatic stellate cell trans-differentiation. J Cell Physiol 2015, 230:1075-1085

18. Ramani K, Yang H, Kuhlenkamp J, Tomasi L, Tsukamoto H, Mato JM, Lu SC: Changes in the expression of methionine adenosyltransferase genes and S-adenosylmethionine homeostasis during hepatic stellate cell activation. Hepatology 2010, 51:986-995

19. Norbury C, Blow J, Nurse P: Regulatory phosphorylation of the p34cdc2 protein kinase in vertebrates. EMBO J 1991, 10:3321-3329

20. Nacerddine K, Lehembre F, Bhaumik M, Artus J, Cohen-Tannoudji M, Babinet C, Pandolfi PP, Dejean A: The SUMO pathway is essential for nuclear integrity and chromosome segregation in mice. Dev Cell 2005, 9:769-779

21. Tahmasebi S, Ghorbani M, Savage P, Gocevski G, Yang XJ: The SUMO conjugating enzyme Ubc9 is required for inducing and maintaining stem cell pluripotency. Stem Cells 2014, 32:1012-1020

22. Mo YY, Yu Y, Theodosiou E, Ee PL, Beck WT: A role for Ubc9 in tumorigenesis. Oncogene 2005, 24:2677-2683 